\title{
An audit- indications and diagnosis of bone marrow biopsies at a tertiary care hospital in Saudi Arabia
}

\begin{abstract}
Objective: We conducted the study to observe the common indications of bone marrow biopsy and frequencies of various disorders diagnosed on bone marrow examination in our center.

Materials and methods: It was a descriptive retrospective audit conducted at the Division of Hematology, Department of Pathology at King Khalid University Hospital in Riyadh. All bone marrow biopsies performed and reported from January 2014 till December 2015 on patients of all age groups and both genders were analyzed.

Results: Total 481 bone marrows were examined during this two years' time period. All relevant information were extracted and analyzed including reasons of referral and provisional diagnosis made by clinicians. Ages ranged from 5 weeks to 99 years old, and male to female ratio 1.4:1. The common indication of bone marrow biopsy is for diagnosis and management of acute leukemia, then for staging of lymphoma and work up of pancytopenia. Acute leukemia followed by myeloproliferative diseases was most frequently diagnosed malignant disorders and idiopathic thrombocytopenic purpura was common benign hematological finding on bone marrow examination.

Conclusion: Bone marrow examination is a useful tool in ascertaining diagnosis of various malignant hematological disorders. Most common indication of the procedure in our hospital was for diagnosis and management of acute leukemia and as well, on bone marrow examination acute leukemia was frequently diagnosed malignant hematological disease. The referrals of bone marrow biopsy for anemia or nonhematological disorders were less commonly observed from our center.
\end{abstract}

Keywords: bone marrow, indication, frequency, referrals, hematological disorder, non-hematological disorders
Volume 6 Issue 5 - 2018

\author{
Fatma Said Al Qahtani, Naveen Naz Syed \\ Department of Pathology, King Khalid University Hospital, \\ Kingdom of Saudi Arabia
}

\begin{abstract}
Correspondence: Fatma Said Al Qahtani, MBBS, KSUFpath, Assistant Professor and Consultant Hematopathologist, Division of Hematology, Department of Pathology, College of Medicine, King Saud University, King Khalid University Hospital, Riyadh, Kingdom of Saudi Arabia, Tel: 00966-56807499I, Email naveen.aberi@gmail.com
\end{abstract}

Received: August 26, 2018| Published: October 17, 2018

\section{Introduction}

Bone marrow aspirate and trephine examination has a significant role in diagnosis, staging and management of malignant hematological disorders. ${ }^{1}$ In addition, it is a valuable diagnostic tool in number of non-hematological diseases or systemic illness like pyrexia of unknown origin (PUO), storage disorders, infectious diseases including Leishmaniasis, or granulomatous lesions ${ }^{1-4}$ and metastatic solid tumors. ${ }^{1,5,6}$ Sometimes, systemic illness or non-hematological disorders can mimic sign and symptoms of hematological diseases, if patient presents with pallor, bleeding, lymphadenopathy, or hepatosplenomegay. In such cases bone marrow examination may either confirm the suspected diagnosis or direct a clue to unsuspected systemic or non-hematological disease. ${ }^{4,7}$ Sometimes cases referred by clinicians with some provisional diagnosis but found to have some other diseases on bone marrow biopsy.

Bone marrow aspirate is a reliable tool for evaluation of cellular morphology and trephine provides detailed information about bone marrow cellularity, bone architecture, overall hematopoiesis and post chemotherapy changes. ${ }^{6,8}$

The objective of this study was to find out the common indications and bone marrow examination findings at tertiary care university hospital. In addition, to ascertain role of this procedure in the diagnosis of non-hematological or benign hematological diseases in our setup. These results might be helpful for clinicians in selecting cases of bone marrow biopsy as numbers of patients are referred by them in a tertiary care hospital.

\section{Materials and methods}

It was a descriptive retrospective audit conducted over a period of two years from January $1^{\text {st }} 2014$ till December $31^{\text {st }} 2015$, at the Section of Hematology, Department of Pathology, King Khalid University Hospital in Riyadh, Saudi Arabia. All patients underwent bone marrow biopsy either admitted in clinical wards or referred from outpatient clinics at the King Khalid Hospital. We analyzed 481 bone marrow aspirate and trephines in all age groups and both gender.

Patient age, sex, clinical history, indication for the procedure and provisional diagnosis made by primary clinicians were recorded. After routine hematological investigations, bone marrow specimens were obtained from the posterior iliac crest in all patients according to standard technique. ${ }^{6,8}$

Peripheral blood and bone marrow smears were prepared and stained by wright-Giemsa stain while trephine were decalcified and paraffin embedded blocks were stained with usual haematoxylin and eosin (H\&E) stain and examined. Appropriate marrow immunohistochemical, and reticulin stains were used where necessary.

Descriptive statistical analysis of the data was performed by using SPSS software (version 22.0, SPSS, Chicago, Illinois, USA) to determine the frequencies with percentages of various diseases involving the bone marrows and to elaborate the indications of the procedure.

\section{Results}

During the study period, a total of 481 bone marrow biopsies were 
performed and evaluated. There were 281 males and 200 females, male to female ratio 1.4:1. The patient's ages ranged from 5 weeks to 99 years, with a mean age of 38years ( $\mathrm{SD} \pm 2.33)$. Children $(<15$ years $)$ were $92(19.1 \%)$ and $389(80.9 \%)$ bone marrow biopsies from adults.

As it is a tertiary care hospital, three hundred and fifty five (73.8\%) patients were referred for bone marrow examination by the clinicians with a provisional diagnosis based on their clinical presentations or initial laboratory work up. Of these, 292 patients were already admitted at clinical wards of the hospital and 63 were referred from outpatient clinics. Other 126 bone marrow specimens were performed from patients of hematology/oncology wards or clinics.

The frequent indications or referrals of bone marrow examination were as follows: 89 patients had a procedure for diagnosis or management of acute leukemia, 75 for staging of lymphoma and in 63 cases for work up of pancytopenia (Table 1).

Table I Indications for bone marrow biopsy at King Khalid University Hospital, Riyadh

\begin{tabular}{ll}
\hline Indications of bone marrow examination & $\mathbf{n = 4 8 ~ I ~}$ \\
\hline Diagnosis and management of acute leukemia & $89(18.5 \%)$ \\
Staging of lymphoma & $75(15.5 \%)$ \\
Pancytopenia & $63(13.09 \%)$ \\
Anemia/ pallor & $40(8 \%)$ \\
Hepatosplenomegaly & $36(7.4 \%)$ \\
PUO & $36(7.4 \%)$ \\
Bleeding/ thrombocytopenia & $32(6.6 \%)$ \\
Lymphadenopathy & $31(6.4 \%)$ \\
Leucoerythroblastic picture on blood smear & $16(3.3 \%)$ \\
Weakness & $16(3.3 \%)$ \\
Leukocytosis & $16(3.3 \%)$ \\
Weight loss & $14(2.9 \%)$ \\
Neutropenia & $10(2 \%)$ \\
Abdominal or pelvic mass & $10(2 \%)$ \\
\hline
\end{tabular}

\section{Bone marrow examination findings}

Of the 481 bone marrows examined, 22 biopsies were "inadequate or not suitable" for assessment due to blood clot, crushed, or dilute specimen. 207 (43\%) bone marrows reported as normal showing active tri-lineage hematopoiesis, and 17 trephines showed mild to moderate hypo-cellular changes as compared to the patient's respective age with no other significant findings, most of these bone marrows were performed in follow-up cases receiving therapy to see remission or response or for work up of pancytopenia.

Remaining 235 (48.8\%) bone marrows showed conclusive diagnostic findings. Among these 226 (96\%) bone marrows reported with hematological diseases, malignant hematological disorders were seen in $194 / 235(82.5 \%)$, and $32 / 235(13.5 \%)$ had non-malignant hematological conditions. Remaining 9 (4\%) bone marrow biopsies were involved with non-hematological diseases.

Commonest bone marrow examination finding was acute leukemia in 72 out of $481(15 \%)$ bone marrows examined and was the most common hematological malignancy, followed by $50(10.3 \%)$ cases diagnosed as myeloproliferative disorders and $23(4.7 \%)$ had multiple myeloma. In non-malignant hematological conditions, 17/481(3.5\%) had idiopathic thrombocytopenic purpura (ITP) followed by anemia in $15(3.1 \%)$ including (megaloblastic anemia in 5, iron deficiency anemia (IDA) in 4 patients).

Non-hematological diseases reported in $9(1.8 \%)$ out of 481 bone marrow trephines. Six bone marrows were infiltrated with metastatic tumors; three of these had neuroblastoma in children. Bone marrow examination in three adults revealed metastatic adenocarcinoma from prostate, breast and bladder respectively. All of these cases were diagnosed on bone trephines with help of a panel of immunohistochemical stains. Other three bone marrows showed granulomatous changes, leishmaniasis and Neimann Pick disease respectively.

Table 2 Showing spectrum and frequencies of various hematological and non-hematological disorders reported on bone marrow examination at Department of Hematology at King Khalid University Hospital during 2009 to 2010.

Table 2 Frequencies of various disorders on bone marrow examination, split in three groups as follows
A. Shows frequency of malignant hematological disorders
B. Shows frequency of non-hematological disorders on bone marrow
C. Shows frequency of benign hematological disorders

\begin{tabular}{|c|c|c|}
\hline A. Malignant Hematological Disorders & $N=|94 / 48|$ & Percentage \\
\hline Acute leukemia & 72 & \multirow{2}{*}{$15 \%$} \\
\hline AML/ ALL/ Bi-phenotypic & $41 / 26 / 5$ & \\
\hline Myeloproliferative disorder & 50 & \\
\hline Chronic Myeloid Leukemia & 27 (Chronic phase 22, accelerated 2 and blast 3 ) & \\
\hline Essential Thrombocythemia & 13 & $10.30 \%$ \\
\hline Polycythemia & 5 & \\
\hline Myelofibrosis & 5 & \\
\hline Multiple myeloma & 23 & $4.70 \%$ \\
\hline Lymphoma & 18 & \\
\hline Non-Hodgkins Lymphoma & $14 / 50$ & $3.70 \%$ \\
\hline Hodgkins Lymphoma & $25 / 4$ & \\
\hline
\end{tabular}


Table Continued....

\begin{tabular}{lll}
\hline A. Malignant Hematological Disorders & $\mathrm{N}=\mathbf{1 9 4 / 4 8} \mathbf{I}$ & Percentage \\
\hline Chronic lymphocytic leukemia & 17 & $3.50 \%$ \\
Myelodysplasia & 12 & $2.50 \%$ \\
Hairy cell leukemia & 2 & $0.40 \%$ \\
Total & 194 & \\
\hline
\end{tabular}

\begin{tabular}{lll}
\hline B. Non-Hematological Diseases & N=9/48 I & Percentage \\
\hline Metastatic tumor & 6 & $1.20 \%$ \\
Storage disorder Neimann Pick disease & I & $0.20 \%$ \\
Leishmaniasis & I & $0.20 \%$ \\
Granulomatous changes & I & $0.20 \%$ \\
Total & 9 & \\
\hline
\end{tabular}

\begin{tabular}{lll}
\hline C. Non-Malignant Hematological disorders & N=32/48 I & Percentage \\
\hline Idiopathic thrombocytopenic purpura (ITP) & 17 & $3.50 \%$ \\
Anemias & 15 & \\
Megaloblastic anemia & 5 & \\
Iron deficiency anemia (IDA) & 4 & $3.10 \%$ \\
IDA+ITP & 3 & \\
Autoimmune hemolytic anemia (AIHA) & $\mathrm{I}$ & \\
Congenital dyserythropoietic anemia (CDA) & $\mathrm{I}$ & \\
Anemia of chronic disease (ACD) & $\mathrm{I}$ & \\
Total & 32 & \\
\hline
\end{tabular}

\section{Discussion}

Examination of bone marrow aspirate and trephine is a valuable tool in diagnosing a suspected disease or in assessing a known case of hematological disorders and also in evaluation of patients with nonhematological diseases or systemic illness. ${ }^{1,7,9,10}$

This study was conducted to determine the major indications and frequencies of various disorders diagnosed on bone marrow aspirate and trephines at tertiary care university hospital. We reported variable spectrum of diseases on bone marrow examination in our cohort of patients. This study showed that acute leukemia was the most commonly encountered indication, and bone marrow examination finding in our setup, an observation similar to others from Kingdom of Saudi Arabia (KSA). ${ }^{11,12}$

Second frequent reason of bone marrow referrals was for staging of lymphoma. It was also already reported common indication and malignancy in local reports. ${ }^{11,12}$ We evaluated 50 specimens for staging of non-Hodgkin's lymphoma (NHL) and 14 showed bone marrow infiltration, mostly with large B cell lymphoma. 25 trephines were examined for Hodgkin's lymphoma involvement and only 4 were found to have stage IV disease.

Pancytopenia is one of the diagnostic dilemmas to clinicians and here third common reason of bone marrow referrals. Among 63 patients presented with pancytopenia, 27 showed normocellular bone marrow with active tri-lineage hematopoiesis suggesting cause of pancytopenia is peripheral destruction, Aplastic anemia or hypocellular trephines were reported in five cases suggestive of idiopathic or drug induced changes. Table 3, Enlist the causes of pancytopenia in these 63 patients.

Table 3 Bone marrow examination findings in cases referred for work up of pancytopenia

\begin{tabular}{ll}
\hline Pancytopenia & $\mathbf{N}=63$ \\
\hline Normal bone marrow & $27(42 \%)$ \\
Acute leukemia & $07(11 \%)$ \\
Myelodysplasia & $07(11 \%)$ \\
Aplastic anemia & $05(7.9 \%)$ \\
Inadequate specimen for reporting & $05(7.9 \%)$ \\
Megaloblastic anemia & $04(6.3 \%)$ \\
Myelofibrosis & $04(6.3 \%)$ \\
Hairy cell leukemia & $02(3.1 \%)$ \\
Leishmaniasis & $01(1.5 \%)$ \\
Metastatic tumor & $01(1.5 \%)$ \\
Total & 63 \\
\hline
\end{tabular}

We found higher numbers of bone marrows involved with malignant hematological disorders than benign or non-hematological diseases. Various studies from other regions revealed an incidence of benign hematological disorders in bone marrows from $60-80 \% \%^{2,9,10}$ and this can be explained by prevalence of anemia in their regions. 
Acute leukemia was the common indication, and bone marrow examination finding in our setup and as well from other centers in Kingdom. ${ }^{11,12}$ However, in this report acute myeloid leukemia (AML) is more frequently encountered than acute lymphoblastic leukemia (ALL) and it may be because our study population comprised of more adult patients.

Next to this, other frequently diagnosed hematological malignancies were myeloproliferative disorder (MPD) followed by multiple myeloma (MM), variable incidence rates were reported in regional reports. ${ }^{11,13}$ Among 50 patients of MPD, 27 were of chronic myeloid leukemia (CML), 13 had essential thrombocythemia (ET), 5 cases of polycythemia rubra vera (PRV) and primary myelofibrosis (MF) respectively. Patients diagnosed with these disorders are mainly adults $>45$ years old. Incidence of myeloproliferative disorder and myeloma increases with the age and rare in childre ${ }^{1,3}$ so the higher incidence of these disorders in this study is because of $80 \%$ study population were adult patients in our group.

In non-malignant hematological diseases, idiopathic thrombocytopenic purpura (ITP) was common, diagnosed mainly in children and results are comparable with other reports from KSA. ${ }^{11}$ We reported very small number of bone marrows that were involved with non- hematological disorders and were incomparable with others.

Although contrary to other. ${ }^{2,9,10}$ we found that bone marrow involved less often with benign hematological and non-hematological disorders. These differences in bone marrow examination findings may be because of our sample size, or study population involving more adults as benign disorders like ITP frequently occur in children, and or we received less referral for workup of anemia. In addition most cases referred by the clinicians who can utilized easily available imaging techniques along with tissue biopsy in a tertiary care hospital for diagnosis of metastatic tumors. These reasons can decrease the yield of bone marrow examination for diagnosis of benign and nonhematological diseases in our center.

In $4.5 \%$ bone marrow biopsies, sample was inadequate for reporting, and was mostly in children. The reported failure rates of bone marrow biopsy range from $2-10 \%$ or even higher. ${ }^{2,11}$ Bone marrow biopsy is an uncomfortable procedure for the patients and in a tertiary care centers it should be performed by trained person to minimize the inadequacy of specimens.

Almost half of the bone marrow specimens were reported normal but most of these were done in known patients with hematological diseases to assess response of treatment, an observation similar to others. $^{11}$

Herein; interesting finding is, bone marrow biopsy performed for evaluation of anemia is one of the most frequent indication in $20-50 \%$ biopsies by multiple studies from different parts of the world. $2,4,5,7,9,10$ In contrast to these, we found only $8 \%$ bone marrows were referred for workup of anemia, concordance with another study in Kingdom. ${ }^{11}$ We found, 3\% bone marrow biopsies reported with variable types of anemia, which is similar to a local report ${ }^{11}$ and few others. ${ }^{14}$

Nevertheless, in contrast to our findings; nutritional anemia reported as common bone marrow examination findings by some. ${ }^{2,67,7,9,10}$ These differences can be explained by nutritional deficiencies or $H$. pylori infections are less common in our region. In addition, we conclude that bone marrow examination had not utilized as diagnostic tool for anemia in our hospital unless other relevant blood investigations failed to suggest the cause. So, indication of bone marrow examination for workup of anemia is not common here as majority of patients can be easily diagnosed on peripheral blood smears or other easily available blood tests and treated as outpatients.

\section{Conclusion}

Herein, indications of bone marrow examination were for evaluation of acute leukemia and lymphoma. The yield of bone marrow examination for diagnosis of anemia or other benign or nonhematological disorders are very low. Common diagnosis encountered in our setting was acute leukemia and myeloproliferative disorders. Bone marrow examination is an important tool for diagnosis and assessing response of therapy in malignant hematological disorders.

\section{Acknowledgements}

The authors would like to thank Deanship of Scientific Research King Saud University for supporting this study.

\section{Authorship criteria and contribution}

This manuscript has been read and approved by all the authors, the requirements for authorship have been met, and we believe that the manuscript represents honest work. All authors are involved in design of study or acquisition, analysis and interpretation of data, literature search, drafting the article, manuscript preparation or revising it critically for important intellectual content; and final approval of the version to be published.

\section{Conflict of interest}

The authors of this paper have no conflicts of interest, including specific financial interests, relationships, and/or affiliations relevant to the subject matter or materials included.

\section{References}

1. Syed NN, Moiz B, Adil SN, et al. Diagnostic importance of bone marrow examination in non-hematological disorders. J Pak Med Assoc. 2007;57(3):123-125.

2. Okinda NA, Riyat MS. Bone marrow examination findings at aga Khan University Hospital, Nairobi. East Afr Med J. 2010;87(1):4-8.

3. Manion EM, Rosenthal NS. Bone marrow biopsies in patients 85 years or older. Am J Clin Pathol. 2008;130(5):832-835.

4. Kaur M, Singh Rana AP, Kapoor S, et al. Diagnostic value of bone marrow aspiration and biopsy in routine hematology practice. $J$ Clin Diagn Res. 2014;8(8):FC13-FC16.

5. Bedu-Addo G, Ampem Amoako Y, Bates I. The role of bone marrow aspirate and trephine samples in haematological diagnoses in patients referred to a teaching hospital in Ghana. Ghana Med J. 2013;47(2):74-78.

6. Goyal S, Singh UR, Rusia U. Comparative evaluation of bone marrow aspirate with trephine biopsy in hematological disorders and determination of optimum trephine length in lymphoma infiltration. Mediterr J Hematol Infect Dis. 2014;6(1):e2014002.

7. Parajuli S, Tuladhar A. Correlation of bone marrow aspiration and biopsy findings in diagnosing hematological disorders - a study of 89 cases. Journal of Pathology of Nepal. 2014;4(7):534-538.

8. Bain BJ. Bone marrow trephine biopsy. $J$ Clin Pathol. 2001;54(10):737-742.

9. Gandapur AS, Nadeem S, Riaz M, et al. Diagnostic Importance of Bone Marrow Examination in Haematological Malignant and Non-Malignant Disorders. J Ayub Med Coll Abbottabad. 2015;27(3):692-694. 
10. Shaheen N, Khan MQA, Azim W, et al. Bone marrow aspiration: The diagnostic tool in haematological and non-haematological disorders. Pak J Pathol. 2010;21(1):1-4.

11. Bashawri LA. Bone marrow examination. Indications and diagnostic value. Saudi Med J. 2002;23(2):191-196.

12. Tandon P, Pathak VP, Zaheer A, et al. Cancer in the Gizan province of Saudi Arabia: An eleven year study. Ann Saudi Med. 1995;15(1):14-20.
13. Al Gwaiz LA. Analysis of 3494 bone marrow examination in a referral hospital: indication and interpretations. Saudi Med J. 1997;18:144-147.

14. Hyun BH. Bone marrow examination: Adventures in Diagnostic Hematology. Yonsei Med J. 1986;27(2):100-105. 